\title{
Multi-Conjugate Adaptive Optics with Laser Guide Stars
}

\author{
R.I. Davies ${ }^{1}$, D. Bonaccini ${ }^{2}$, S. Rabien ${ }^{1}$, W. Hackenberg ${ }^{2}$, T. Ott ${ }^{1}$, S. Hippler ${ }^{3}$, \\ U. Neumann ${ }^{3}$, M. Barden ${ }^{1}$, M. Lehnert ${ }^{1}$, F. Eisenhauer ${ }^{1}$, and R. Genzel ${ }^{1}$ \\ 1 Max-Planck-Institut für extraterrestrische Physik, 85741 Garching, Germany \\ 2 European Southern Observatory, 85748 Garching, Germany \\ 3 Max-Planck-Institut für Astronomie, 69117 Heidelberg, Germany
}

\begin{abstract}
The Laser Guide Star Facility for the VLT will be commissioned during 2003. This, of course, can only be the first step towards enhancing the scientific output of the VLT through adaptive optics (AO). So in this contribution we propose the development of a laser guide star (LGS) multi-conjugate adaptive optics (MCAO) system. We consider geometries for the adaptive optics system, and discuss the technology needed to project up to 5 laser beacons. The rationale for such a project is provided by an outline of two primary science drivers.
\end{abstract}

\section{Introduction}

The progress that has been made in understanding advanced adaptive optics techniques in recent years now allows us to design systems that are tailored to particular astronomical requirements. For a limited amount of light from one or more reference sources, this is effectively a trade off between finely sampling (spatially and temporally) the atmospheric turbulence over a small area to obtain extremely high Strehl ratios over a limited field of view, or broadly sampling the turbulence over a large area to obtain moderate performance over a wide field.

Here we discuss which characteristics might be desirable in a system that would have the widest possible applications for $1-2.5 \mu \mathrm{m}$ observations. We argue that laser guide stars are mandatory if these are to be achieved. The particular science drivers we consider - observational cosmologogy and the initial mass function (IMF) in massive galactic star clusters - indicate the potential wealth which can be reaped with LGS MCAO.

\section{MCAO characteristics}

What an astronomer really wants from $\mathrm{AO}$, and which in fact can only be delivered by a MCAO system, can be summarised simply as: obtaining, with high sky coverage, a uniform high Strehl over a large field of view, that field being the same at all wavelengths. Considering each of the points in turn:

- High Strehl ratio is the raison d'être for AO systems, to provide better sensitivity and resolution. The problem of limited Strehl with a single LGS (the cone effect) is solved with MCAO. Strehl ratios greater than $50 \%$ in the $\mathrm{K}$-band, or equivalent in other bands, should be achievable. 
- Uniform Strehl ratio over a large field of view $\left(\sim 1^{\prime}\right.$ can be corrected with relatively few guide stars) is the primary reason for using MCAO, solving the isoplanatic limitation of normal AO. It assures reliable photometry (eg for star clusters or extended objects) and uniform sensitivity (eg for number counts); and a PSF reference would usually be found in the field.

- The same field of view at all wavelengths comes by default with MCAO: the field of view attained is due to the height of the atmospheric turbulence rather than the $\lambda$-dependent isoplanatic angle. For multi-colour studies, properties of the same objects can be measured in different bands.

- High sky coverage ensures that as many programmes as possible can be executed, and is necessary if MCAO is to have an impact on astrophysics.

\section{Natural Guide Stars}

The preferred option for MCAO in terms of cost and complexity would certainly be to use NGS if the requirements above could be fulfilled. We briefly consider the implications of this on the two designs for MCAO that have been proposed.

\subsection{Classical MCAO}

The original MCAO concept involves sensing multiple guide stars separately to provide measurements of the total wavefront aberrations in several directions. By making some assumptions (or additional measurements) about the height and number of turbulent layers, it is possible to derive the phase shifts induced by each. It is then possible to correct these with a DM conjugated to each layer.

The advantage of this method is that relatively few guide stars are needed, typically $3-5$ to correct $\sim 1^{\prime}$. However, only the area between the stars is corrected well, and then only if they are less than $30-60^{\prime \prime}$ apart. Furthermore, the maximum Strehl ratio towards any given guide star depends on its magnitude. A uniform high Strehl can therefore be achieved for only a very few sources. In the vast majority of other possible targets - which is still limited - the Strehl ratio will vary considerably across the field. As a consequence of compromising the performance in this way, the resolution and sensitivity will vary greatly and the data analysis become extremely difficult, losing the advantage that MCAO should have over standard AO.

\subsection{Layer oriented MCAO}

An alternative concept (Ragazzoni et al. 2000) avoids these problems to some extent by using the light from many stars in the field rather than just the few brighter ones. The design uses pyramid wavefront sensors, which allow the light from all the sources to be combined before being sensed. Each of 2-3 turbulent layers is corrected independently and requires one DM and one detector, both of which are conjugated to it. This vastly simplifies the computations, but if many stars are used the optics in the AO system becomes rather more complex. 
Additionally, the light from all the stars in a field is typically only $14-15 \mathrm{mag}$, rather less than that needed for optimal correction (eg NAOS requires stars of $\sim 12$ mag for best performance, Rousset et al. 2000). If on the other hand fewer stars are used, then the problems of variable Strehl across the field return due to strong variations in the signal-to-noise with position.

\section{Laser Guide Stars}

One solution to the problems outlined above is to use artifical guide stars. This applies to either of the concepts mentioned, or even new ones - indeed, one scheme optimised for use with LGS is currently under study. Sodium laser guide stars (and also Rayleigh beacons, although these are not considered here because there are additional MCAO design issues which require further development) allow one to have regularly placed bright sources of the same magnitude. The power and positioning can be adjusted to produce the required uniform high strehl (Berkefeld et al 2001). Simulations by Rigaut et al. (2000) for the Gemini LGS-MCAO system indicate that with 5 LGSs the Strehl ratio varies by only a few percent from the peak value of $60-70 \%$ (at K) over a field of $1-2^{\prime}$ at zenith.

The VLT Laser Guide Star Facility (LGSF) Launch Telescope is already designed to cope with 5 lasers, and is diffraction limited over a radius of $>1^{\prime}$. The pointing of each beam is controlled by a piezo system, allowing them to be moved in a centred square geometry anywhere within this region.

A number of objections to sodium LGS have been raised. We show below that these are not a major hindrance to the implementation of LGS MCAO.

1) The first generation of LGS on 3-4-m telescopes were difficult and inefficient to use, but experience with such systems as ALFA (Hippler et al. 2000, Davies et al. 2000) will lead to significant improvements for LGS at 8-10-m telescopes. 2) Up to 3 NGS are needed to sense the low order modes (the inability to sense tip-tilt from a LGS projects into astigmatism and focus for MCAO). However, the requirements on magnitude and position are far less stringent than before and do not impose strong constraints on sky coverage.

3) The LGS elongation is less than $1^{\prime \prime}$ as long as the launch telescope is behind the VLT secondary. It depends primarily on the horizontal separation of the launch and detection points, which need only be 4-m for an 8-m telescope.

4) Light scattered in the Rayleigh cone interferes with the wavefront sensing. A simple but wasteful option is to pulse a continuous wave laser via extra-cavity modulation (eg as was done on much faster timescales for LIDAR on ALFA, Butler et al. 2000). Alternatively, careful baffling of the Rayleigh light at its focal plane in the AO system may alleviate much of the problem without greatly compromising the brightness or shape of the LGS.

\subsection{Laser Power}

In order for a LGS to provide $10^{6} \mathrm{ph} \mathrm{m}^{-2} \mathrm{~s}^{-1}$ (equivalent) at the Nasmyth focus even in 'poor' conditions, a laser power of $10 \mathrm{~W}$ is needed. This scales to $50 \mathrm{~W}$ 
for MCAO with 5 lasers. As a result ESO is currently pursuing a research programme to develop $589 \mathrm{~nm}$ continuous wave fibre lasers, each producing $10 \mathrm{~W}$ with a $500 \mathrm{MHz}$ bandwidth matched to the mesospheric sodium absorption profile. These systems would be relatively simple to operate and require no additional beam relay since the fibre laser itself could extend to the launch telescope.

A short-term alternative would be to upgrade PARSEC, the laser which will initially be used in the LGSF. PARSEC is a ring dye laser system: pump lasers emitting green $532 \mathrm{~nm}$ light excite molecules in a high speed dye jet, which decay to produce the $589 \mathrm{~nm}$ sodium line emission. It has a master laser operating at low power which can be locked to the right frequency and tuned to produce a high quality output beam. This seeds an amplifier which provides a scalable boost to the final output power. Although a full upgrade could in principle provide $50 \mathrm{~W}$, a very simple change that involves only pumping the existing dye jets with more power, could increase the $589 \mathrm{~nm}$ output to around $25 \mathrm{~W}$.

In this case each LGS would be $\sim 0.8$ mag fainter. Such a loss does not compromise AO performance much and, during most observing, would be recovered: 1) The $10 \mathrm{~W}$ specification for each LGS takes into account observing at an airmass of 2; at an airmass of 1.5, where the seeing is anyway better, each LGS would be $0.3 \mathrm{mag}$ brighter.

2) A sodium column density above $3 \times 10^{13} \mathrm{~m}^{-2}$ (which occurs more than $60 \%$ of the time, Ge et al. 1998) rather than the minimum specified $2 \times 10^{13} \mathrm{~m}^{-2}$ (which occurs $80 \%$ of the time), would gain another $0.45 \mathrm{mag}$.

3) At least $0.1 \mathrm{mag}$ is expected to be gained through advances in the fibre beam relay system for PARSEC.

\section{Science Cases}

\subsection{Observational Cosmology}

With current AO systems, the only way to proceed with observational cosmology is to study objects close to bright stars: within $\sim 30^{\prime \prime}$ for the K-band, or $\sim 15^{\prime \prime}$ in the J-band (to detect $\mathrm{H} \alpha$ in objects at $z \sim 1$ ). The other is to use MCAO.

Observations with ALFA of the $1^{\prime}$ field around such a star, with $30 \% \mathrm{~K}$ band Strehl, have revealed a surprisingly high number of galaxies and faint point sources at $\mathrm{K} \sim 19-20.5$ (Davies et al. 2001), at which magnitude the mean redshift is $z=0.7$ (Cowie et al. 1996). The resolution of $0.15^{\prime \prime}$, already better than that attained with NICMOS on the HST, is sufficient to easily measure the light profiles, one of the robust parameters used for the classification of high redshift objects in studies of the cosmological evolution of galaxies (Abraham 1999). Simulations indicate that a 1 hour integration on the VLT with $50 \%$ Strehl in the K-band is sufficient to see the more detailed morphology of K 19 galaxies, as shown in Fig. 1. As a result of the difference between observed and rest frames, such data would be far more successful at classification of high redshift objects than the similar resolution HST I-band data, for which at $z=0.9$ about $24 \%$ of spirals are misclassified as peculiars (Brinchmann et al. 1998). 


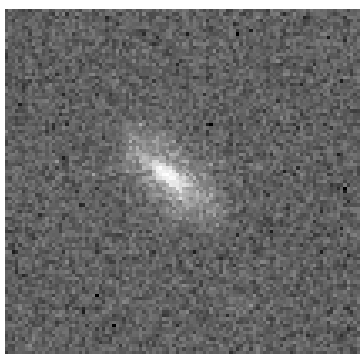

Fig. 1. $5^{\prime \prime} \times 5^{\prime \prime}$ simulation of a 1 hr integration on the VLT with a $50 \%$ Strehl showing a $\mathrm{K}=19$ galaxy, in this case NGC 253 redshifted to $z \sim 1$. The fainter extended emission is clearly seen, allowing a morphological classification.

Another aspect of high redshift work is the Tully-Fisher relation, which correlates the rotational velocity of a galaxy's disk with its absolute magnitude. Changes in the relation at higher redshifts (eg Barden \& Lehnert, priv. comm.) clearly indicate evolution in the mass to light ratio of disks, but whether this is purely passive evolution or indicates something more requires careful analysis of galaxy evolution models. This type of work is extremely difficult with ground-based telescopes: the galaxies are typically $1^{\prime \prime}$ across, and so even at good observing sites it is not only difficult to align a slit along the major axis but the rotation curves are barely resolved. AO would have 2 benefits. The first is to reduce the blending in the rotation curve by putting the light back where it should be, without necessarily increasing the surface brightness. The second is effectively to increase the surface brightness, because the (clusters of) HII regions responsible for the emission lines appear as point sources at the diffraction limit of the VLT as shown in Fig. 2. For such work, it is undesirable and unnecessary
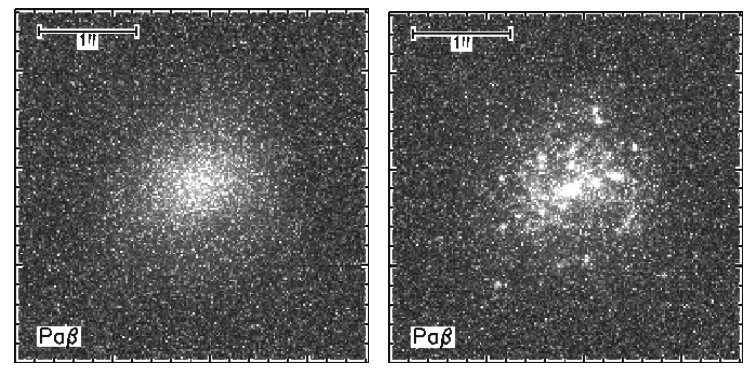

Fig. 2. $\mathrm{H} \alpha$ line map of IC 342 as it would appear at $z=0.7$ with the $\mathrm{Pa} \beta$ line imaged in the K-band. Left is seeing limited; right is with a $50 \%$ Strehl, showing that the HII regions are clustered on scales matched to the resolution of the VLT.

to fully sample the PSF: the former because the sensitivity would be limited by read noise and dark current, the latter because only a few independent points are needed to measure the rotation curve. Infact, pixels of $\sim 0.075^{\prime \prime}$ are ideal, equivalent to $\sim 500 \mathrm{pc}$ at $z=1$. The pixel gain (ie increase in flux that should be in a pixel, compared to the seeing limited case) is $\times 25$ for $50 \%$ Strehl in the K-band or, for a similar AO performance, $\times 15$ for $18 \%$ Strehl in the J-band. Using MCAO to extend the current work to lower surface brightness galaxies or to 
higher redshifts, and to measure several galaxies simultaneously (with a multiobject spectrograph or integral field unit), would enable important progress in understanding galaxy evolution.

\subsection{Massive Star Clusters}

A rather different application for MCAO is in the study of the IMF in massive star clusters. Using ADONIS, Eisenhauer et al. (1998) reached about $20 \%$ K-band Strehl ratio in the centre of NGC 3606 and were able to measure its IMF from about 800 stars - but only after careful deconvolution using PSFs appropriate to different parts of the cluster. They found that while the IMF above $\sim 30 \mathrm{M}_{\odot}$ was similar to both the Scalo field star IMF and that measured in the Upper Scorpius association, for stars below $\sim 30 \mathrm{M}_{\odot}$ the IMF had a much shallower slope. Later work using the VLT (Brandl et al. 1999) was far more sensitive, but because of the seeing limited resolution of $0.3-0.4^{\prime \prime}$, the uncertainties in correcting for completeness remain too great to permit a final sensus of the low mass stellar population - something that would be possible with MCAO, which could image the central 2-3 pc of the cluster in all of the JHK bands and reach a high Strehl ratio in each.

\section{Conclusion}

We have argued that laser guide stars are essential for MCAO to be scientifically useful to the astronomical community: they provide the only way to achieve a high uniform Strehl over a large field with good sky coverage.

The technical problems in providing sufficient laser power are being addressed. A simple upgrade to PARSEC would provide enough power at least for most observing conditions, and is a realistic option within the next few years.

The two science cases represent areas where MCAO could make important contributions, but which are unattainable without the use of laser guide stars.

\section{References}

1. R. Abraham: Ap\&SS, 269-270, 323 (1999)

2. T. Berkefeld, A. Glindemann, S. Hippler: Exp. Ast., 11, 1 (2001)

3. B. Brandl et al.: A\&AL, 352, 69 (1999)

4. J. Brinchmann et al.: ApJ, 499, 112 (1998)

5. D. Butler et al.: SPIE, 4007, 358 (2000)

6. L. Cowie, A. Songaila, E. Hu, J. Cohen: AJ, 112, 839 (1996)

7. R.I. Davies et al.: Exp. Ast., 10, 103 (2000)

8. R.I. Davies, M. Lehnert, A. Baker, S. Rabien: In Galaxies and their Constituents at the Highest Angular Resolutions, IAU symp. 205, (2001)

9. F. Eisenhauer, A. Quirrenbach, H. Zinnecker, R. Genzel: ApJ, 498, 278

10. J. Ge et al.: SPIE, 3353, 242 (1998)

11. S. Hippler et al.: SPIE, 4007, 95 (2000)

12. R. Ragazzoni, J. Farinato, E. Marchetti: SPIE, 4007, 1076 (2000)

13. F. Rigaut et al.: Internal Report RPT-AO-G0091, Gemini Observatory (2000)

14. G. Rousset et al.: SPIE, 4007, 72 (2000) 